\title{
Circadian waveform bifurcation, but not phase-shifting, leaves cued fear memory intact
}

Harrison $\mathrm{EM}^{\mathrm{a}, \mathrm{b}}$, Carmack $\mathrm{SA}^{\mathrm{c}}$, Block $\mathrm{CL}^{\mathrm{d}}$, Sun $\mathrm{J}^{\mathrm{a}, \mathrm{b}}$, Anagnostaras $\mathrm{SG}^{\mathrm{b}}$, Gorman $\mathrm{MR}^{\mathrm{a}, \mathrm{b}}$

a. Center for Circadian Biology

University of San Diego, California

9500 Gilman Dr.

La Jolla, CA 92093

b. Department of Psychology

University of California, San Diego

9500 Gilman Dr.

La Jolla, CA 92093

c. Neurobiology of Addiction Section

National Institute on Drug Abuse

National Institutes of Health

Baltimore, MD, USA

d. Department of Psychology and Neuroscience

417 Chapel Drive

Duke University

Durham, NC 27708-0086

Corresponding author:

Elizabeth M. Harrison

University of San Diego, California

9500 Gilman Dr.

La Jolla, CA 92093

858-345-0705

emharrison@ucsd.edu

C 2016. This manuscript version is made available under the Elsevier user license 


\begin{abstract}
In mammals, memory acquisition and retrieval can be affected by time of day, as well as by manipulations of the light/dark cycle. Under bifurcation, a manipulation of circadian waveform, two subjective days and nights are experimentally induced in rodents. We examined the effect of bifurcation on Pavlovian fear conditioning, a prominent model of learning and memory. Here we demonstrate that bifurcation of the circadian waveform produces a small deficit in acquisition, but not on retrieval of fear memory. In contrast, repeated phaseshifting in a simulated jet-lag protocol impairs retrieval of memory for cued fear. The results have implications for those attempting to adjust to shift-work or other challenging schedules.
\end{abstract}

Keywords: circadian, waveform, bifurcation, Pavlovian fear conditioning, learning, memory

\title{
1. Introduction
}

In mammals, many aspects of physiology and behavior exhibit circadian, or approximately $24 \mathrm{~h}$, rhythms that are orchestrated by the suprachiasmatic nucleus (SCN), a master pacemaker in the hypothalamus. Alternation between an internal, physiological "subjective" day and night is endogenously generated and persists even in the absence of any light information. Under typical conditions, however, the precise timing of these rhythms is determined by the exogenous signals of daily light schedules. After abrupt schedule changes such 
as travel across time zones, this synchrony between external and internal time is disrupted, and individual internal rhythms may dampen or continue to oscillate robustly but fall out of alignment with one another. Such forms of circadian disruption have negative consequences for health and performance in mammals 1

In a novel entrainment paradigm termed "bifurcation," exposure to a light/dark/light/dark (LDLD) schedule in rodents facilitates a reorganization of the circadian system into two periods of alternating locomotor activity and rest per 24 hours. In addition to behavior, other rhythms that are markers of circadian day and night -- melatonin, light responsiveness and SCN function -- are bimodally expressed in bifurcated animals ${ }^{2-5}$. Bifurcation results in a relatively stable entrainment state that can be rapidly induced ${ }^{4}$, is robust against perturbations of the light/dark schedule ${ }^{6,7}$, and enhances re-entrainment to light/dark schedules ${ }^{8}$. As such, it has been speculated that bifurcation in humans might mitigate some harms of shiftwork or other challenging schedules ${ }^{7}$. Thus, we aimed to assess whether the reorganization of the circadian system observed in bifurcated animals has negative cognitive effects similar to those observed during or after circadian disruption utilizing a simulated jet-lag paradigm.

Behavioral evidence suggests that the twice-daily rhythms observed in bifurcated animals do not represent one $12 \mathrm{~h}$ rhythm, but rather two $24 \mathrm{~h}$ rhythms, both originating from the SCN but oscillating in anti-phase. Within the same animal, activity patterns in each of the two bouts may differ systematically in a number of ways, including the relative amount and/or timing of activity and 
the magnitude of behavioral response to acute light pulses ${ }^{2,9}$, The twice-daily activity pattern is therefore hypothesized to reflect two separate circadian oscillations generated by the circadian system. In fact, preliminary data from SCN core and shell subregions suggest that these two oscillations may each be generated by one of these subregions. Thus, although much of behavior and physiology of bifurcated mice recurs on a $12 \mathrm{~h}$ basis, its underlying clock substrate is organized in terms of $24 \mathrm{~h}$.

Pavlovian fear conditioning is a model of learning and memory well-suited to examine these hypotheses for a number of reasons, including a well-defined neurobiology ${ }^{10-12}$. In Pavlovian fear conditioning, animals are placed in a novel environmental context wherein a tone is paired with a shock. After training, rodents exhibit fear by freezing when returned to the training context or when presented with the tone in a novel context. Contextual and cued fear conditioning are dissociable: contextual fear is a prominent animal model of declarative memory ${ }^{10}$ and evidence suggests it is dependent on both hippocampus and amygdala, whereas cued fear depends on the amygdala ${ }^{12}$. Further, conditioned fear is a paradigm with a relatively short, discrete time course for both training and testing and can therefore be measured during circadian manipulations without disrupting the independent variable of the light/dark schedule.

In rodents and humans, many cognitive tasks show a circadian peak in performance, including acquisition and retrieval of memories ${ }^{13,14}$. Although in many experimental paradigms it is difficult to dissociate the circadian effects of these two stages of memory, a number of recent studies have elegantly 
succeeded in doing so. In Pavlovian fear conditioning, mice trained during the day exhibit more conditioned freezing during acquisition than mice trained in the night, whereas retrieval for both conditioned and cued fear peaked in the day independent of training time ${ }^{15}$. This was the case both when mice were tested in the environmental day (during the light phase), and during the subjective day (during the internal, physiological day programmed by the $\mathrm{SCN}$ in the absence of light/dark cues). In another study, mice trained in the evening exhibited lower rates of contextual freezing at $12 \mathrm{~h}$, but not $24 \mathrm{~h}$, post-training, while mice trained in the morning show no such phase-dependence ${ }^{16}$. In a third paradigm, mice phase-shifted immediately before training performed best 24 , rather than 18 or $32 \mathrm{~h}$ post-training ${ }^{17}$. Taken together, these results may reflect a "time-stamp" for circadian phase (and not environmental time) of retrieval, found previously in hamsters in a conditioned place preference protocol ${ }^{18}$. Circadian rhythms have also been observed in hippocampal long-term potentiation (LTP), a prominent cellular model of learning ${ }^{19,20}$.

It follows, then, that performance on memory tasks is subject to impairment following perturbations of the circadian clock or the light/dark schedule. This has been demonstrated in many ${ }^{17,21-24}$, but not all ${ }^{22,23}$, disruption and memory paradigms. Moreover, arrhythmic Siberian hamsters show deficits in long-term object recognition and spatial learning ${ }^{25,26}$. In addition to behavioral decrements, mice exposed to a 7-h day to which they cannot entrain show decrements in hippocampal LTP ${ }^{21}$, and two recent experiments indicate that chronic phase advances impair hippocampal neurogenesis ${ }^{27,28}$. Finally, 
mutations in core mammalian clock components including Cry and Per can result in learning deficits (for review, see ${ }^{1}$ ). It is unknown to what extent several aspects of circadian disruption contribute to specific learning deficits - e.g., exposure to light during subjective night; reduction in rhythm amplitude or synchrony; repeated phase-shifting, etc. Besides direct clock effects, various downstream physiological processes that are typically under circadian control such as sleep and activity may also contribute to learning and memory deficits 16,29-32. Thus, we may expect changes in learning and memory under bifurcated conditions.

Furthermore, in contrast to effects of shifting the timing, or phase, of circadian rhythms, little is known about the consequences of changing the shape, or waveform, of these rhythms for learning and memory. Natural seasonal variation in circadian waveform (i.e., photoperiodism) results in an extension of subjective night, and there is evidence of altered cognitive and affective outputs in mammals exposed to long winter nights. For example, rats and hamsters exposed to short photoperiods mimicking long winter nights display more depressive and anxiety-like behavior than animals exposed to simulated long summer days ${ }^{33,34}$. Additionally, exposure to long winter nights results in reduced hippocampal volume, decreased hippocampal LTP, and impaired spatial learning and memory in white-footed mice ${ }^{35}$. It is unclear whether or not these observed changes are a direct result of reorganization of the circadian system, however, or instead an indirect effect of seasonal physiological and reproductive changes in the organism induced by the light schedule. Bifurcation enables a steady state 
variation in waveform without the above-mentioned confounding effects inherent in exposure to winter nights.

2. Hypotheses \& Objectives:

In two experiments, we compared Pavlovian fear conditioning in bifurcated and non-bifurcated mice. In Experiment 1, the performance of bifurcated animals was contrasted with that of animals after repeated phase advances in a simulated jet-lag paradigm (Fig 1A). Experiment 2 investigated the contributions of entrainment state, circadian phase of training, and train-test interval (Fig 1B).

\subsection{Hypothesis 1 :}

As bifurcation constitutes a restructuring of circadian organization it may result in impaired retrieval as seen in jet lag or other difficult schedules. Alternatively, because bifurcation is a stable entrainment state, learning impairments seen in other circadian manipulations may be avoided. In experiment 1, bifurcated animals were directly compared to animals that had undergone a simulated jet-lag paradigm (Advancing group). In both experiments, mice from Bifurcated and Control groups were compared 24 hours after training.

\subsection{Hypothesis 2 :}

The two subjective days and nights in bifurcated animals may differentially contribute to learning and memory. Within bifurcated animals, there are a number of reasons to suspect that the two activity/rest bouts observed within one $24 \mathrm{~h}$ 
period might have differential effects on cognition. As discussed above, each of the two subjective days and nights in bifurcated animals are distinct in various ways from the other, including behavior. It may be the case, therefore, that they differentially affect downstream outputs, such as cognition or sleep. Alternatively, we might expect performance in bifurcated animals to have two peaks, one for each of the two subjective days per $24 \mathrm{~h}$. Consequently, in Experiment 2, bifurcated animals trained immediately before the first subjective day were directly compared to animals trained immediately before the second subjective day both $12 \mathrm{~h}$ and $24 \mathrm{~h}$ after training to determine the contribution of each of the distinct $24 \mathrm{~h}$ oscillations.

\section{Materials and Methods}

\subsection{General Methods}

138 C57BL/6J mice aged 5-8 wks were used with approximately equal numbers of males and females balanced across groups. Mice were at least 9 weeks of age at training and were purchased directly from Jackson (West Sacramento, CA; Experiment 1, $\mathrm{n}=42$ ) or bred in house from inbred stock from Jackson (Experiment 2, $\mathrm{n}=96$ ). Mice were group housed $2-5$ per cage at $22 \pm 2^{\circ} \mathrm{C}$ in polypropylene cages $(17.8 \mathrm{~cm} \times 25.4 \mathrm{~cm} \times 15.2 \mathrm{~cm})$ under baseline lighting conditions for two weeks. Unrestricted food and water (Purina Rodent Chow No. 5001, St. Louis, MO) were provided during the entire study. Lighting in the photophase was provided by white tube fluorescent lights providing illumination intensity ranging from 30-100 lux inside individual cages. Bifurcation is typically 
induced under specific, facilitating conditions. These include 1) initial entrainment to LD conditions with a short scotophase; 2) the presentation of a wheel concomitant with introduction to the second daily scotophase; and 3) dim scotophase illumination ( $<0.1$ lux). Bifurcation can take place in the absence of any of these three, but their presence facilitates it ${ }^{36,37}$. The necessity of green versus other spectra of dim light is not yet established. To ensure maximal levels of bifurcation in our study, mice housed under 18:6 baseline conditions were transferred to a 6:6:6:6 light/dark (LD) cycle, with introduction of the wheel coinciding with the new dark period (scotophase; Fig 2B), and scotophases for all groups and conditions were dimly illuminated by green LEDs at an intensity of $<0.1 \mathrm{lux}^{2}$. All experiments were conducted in compliance with the rules and regulations of the Institutional Animal Care and Use Committee, University of California, San Diego.

After two weeks of baseline lighting conditions (12:12 for Control and Advancing animals, 18:6 for animals to be bifurcated), mice were transferred to individual cages with wire running-wheels $(11.4 \mathrm{~cm}$ diameter) in polypropylene cages modified for additional height to accommodate wheel revolutions. Control and Advancing mice remained in 12:12 and were transferred to wheels immediately before lights out (Fig 2A). To induce bifurcation, mice housed under 18:6 baseline conditions were transferred to a 6:6:6:6 light/dark (LD) cycle, with introduction of the wheel coinciding with the new dark period (scotophase; Fig 2B). Upon the transition to individual cages, mice in the Advancing group were exposed to a repeated phase-shifting simulated jet-lag paradigm wherein the 
light schedule was shifted $8 \mathrm{~h}$ earlier (advanced) every 3 days. The first advance coincided with exposure to novel wheels and the last shift was on day 15 of the protocol (Fig 2C).

Locomotor activity rhythms were monitored with a Vitalview data collection system (Minimitter, Bend, OR) that counted the number of electrical closures triggered by a half wheel revolution. Activity counts were compiled into 6-minute bins and entrainment was verified using ClockLab Software (Actimetrics, Wilmette IL).

\subsection{Experimental conditions}

All animals in Experiment 1 were trained within $1.5 \mathrm{~h}$ of the transition to a dark period, and tested $24 \mathrm{~h}$ later (Control, $\mathrm{n}=12$; Bifurcated, $\mathrm{n}=18$; Advancing, $\mathrm{n}=12$ ). The advancing group ended the protocol on the same schedule as the control group and stayed there for one full photocycle before training and subsequent testing $24 \mathrm{~h}$ later (Fig $1 \mathrm{~A}$ and $2 \mathrm{C}$ ).

In Experiment 2, to test the effect of diurnal phase on acquisition, control animals were trained at the beginning of the day ( $\mathrm{AM}, \mathrm{n}=24)$ or 12 hours later at the end of the day (PM, n=24; Fig 1B). While bifurcated animals were likewise trained at the same two time points separated by $12 \mathrm{~h}$, due to the nature of the entrainment state, in both cases training for bifurcated animals took place immediately before a light period/subjective day $\left(\mathrm{AM}_{1}\right.$ or $A M_{2}, \mathrm{n}=24$ for both groups). To test the effect of the consolidation interval, control and bifurcated animals were tested either 12 or $24 \mathrm{~h}$ later ( $\mathrm{n}=12$ for all groups). 


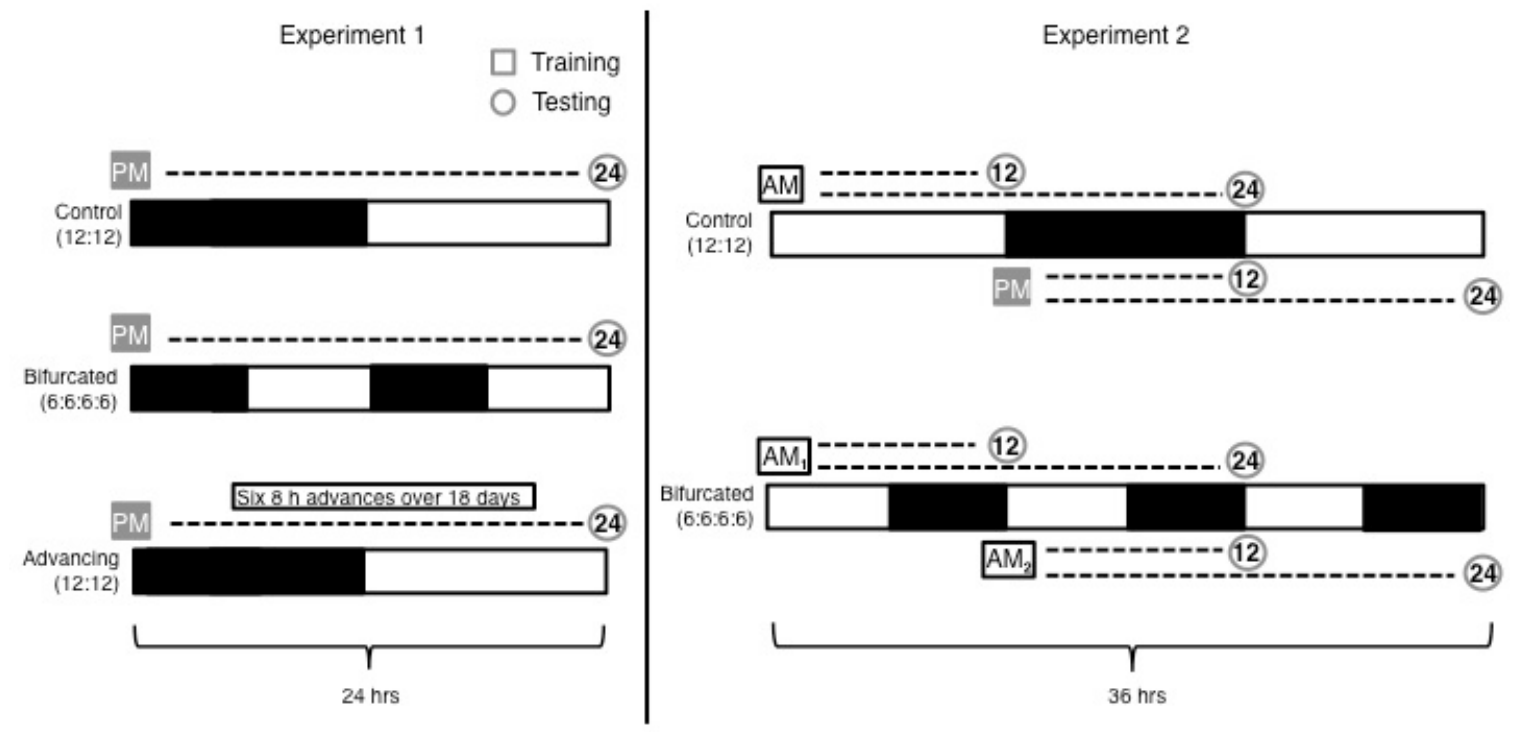

Figure 1. Experimental schema for Experiments 1 (A) and 2 (B). Black and white bars denote the light/dark schedule. In Experiment 1, mice were entrained under 12:12, 6:6:6:6 lighting conditions, or a chronically phase-advancing light schedule. In Experiment 2, mice were entrained under 12:12 or 6:6:6:6 lighting conditions only. All mice were trained and tested within $1.5 \mathrm{~h}$ of a light transition. Boxes labeled " $\mathrm{AM}_{1}$ " or " $\mathrm{AM}_{2}$ " represent training times, whereas circles labeled "12" or "24" represent train-test intervals of 12 or 24 hours, respectively.

\subsection{Fear Conditioning}

All training and testing took place within $1.5 \mathrm{~h}$ of a scheduled light transition.

During lighting transition periods in a 12:12 cycle, animals generally begin to become active and training and testing took place during this time to minimize disruption. Cage changes for all groups occurred one week before training and mice were handled for 5 days prior to training. Four mice were tested concurrently in individual conditioning chambers. Fear conditioning was conducted using the VideoFreeze System (Med-Associates, Inc.). Training and context tests took place in $32 \times 25 \times 25 \mathrm{~cm}$ conditioning chambers encased in 
sound-attenuated boxes and equipped with a speaker in the side wall and a stainless steel grid floor and drop-pan. An overhead LED-based light source provided visible broad spectrum white light. For tone testing trials, chambers were cleaned and scented with a $5 \%$ vinegar solution. White acrylic sheets were placed over the grid floors, a black plastic, triangular teepee was placed inside each box, and near-infrared light created a dark environment. Freezing was automatically scored for each frame $(30 \mathrm{~Hz})$ and cumulated per second by VideoFreeze software as described previously ${ }^{38,39}$.

Training began with a 2-min baseline, followed by three tone-shock pairings at minutes 3,4 and 5 , consisting of a $30-\mathrm{sec}$ tone $(2.8 \mathrm{kHz}, 85 \mathrm{dBA})$ that co-terminated with a 2-sec scrambled, AC constant current foot shock (0.75 mA, RMS). Baseline activity level was measured as the amount activity in the first two minutes of the protocol before any shocks are administered, whereas shock reactivity was measured during the three 2 -sec shocks and averaged. Both measures are expressed in arbitrary units which reflect a noise-corrected number of pixels that changed per second (cumulated @ $30 \mathrm{~Hz}$ ). Animals remained in the training context for an additional 5 min post-shock freezing test. Context testing consisted of returning the animals to the conditioning chamber for a period of 5 min. Tone testing occurred approximately 30-60 min after the context test and consisted of a 2-min baseline, followed by a three 30-sec tone presentations at minutes 3,4 and $5(2.8 \mathrm{kHz}, 85 \mathrm{dBA})$.

\subsection{Statistical Analysis}


Statistical analyses were conducted using SPSS (IBM, New York) and Graphpad Prism (La Jolla CA). In Experiment 1, female mice froze more during acquisition and during the tone test. Sex was therefore covaried in all analyses for Experiment 1. Sex was considered as a factor and had no effect on outcomes for Experiment 2. All tests were evaluated at the alpha $=0.05$ significance level. Greenhouse-Geisser corrections were applied in instances where homogeneity of variance was violated.

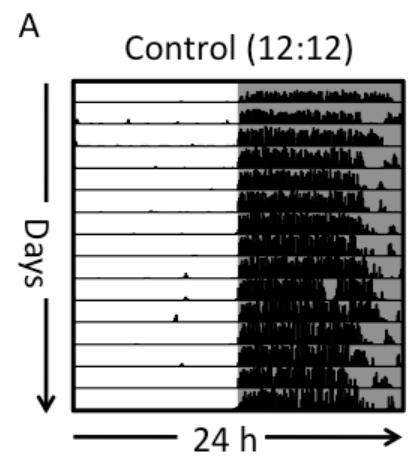

B

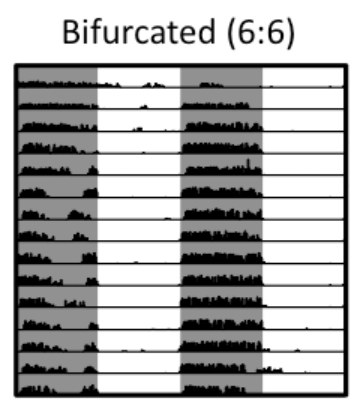

C

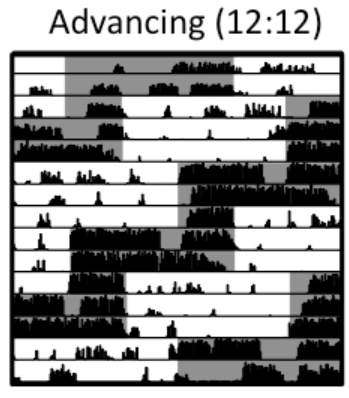

Figure 2. Representative single-plotted actograms from groups in Experiment 1. Wheel-running activity patterns from an animal in 12:12 (A), 6:6:6:6 (B), and the chronically phase-advanced group (C). Actograms are plotted across $24 \mathrm{~h}$ on the $\mathrm{X}$ axis and days on the $\mathrm{Y}$ axis. Gray shading indicates hours of darkness. Note the stability of the bifurcated activity pattern in B, although the two daily dark phases differ in the amount of activity expressed.

\section{Results}

\subsection{Entrainment}

As expected, control animals exhibited activity patterns typical of entrainment to a standard 12:12 light/dark cycle, with wheel-running activity concentrated in the single $12 \mathrm{~h}$ scotophase (Fig 2A). In contrast, within a few days of exposure to the LDLD schedule, most animals in the Bifurcated groups divided their wheel-running activity between the two $6 \mathrm{~h}$ scotophases, albeit 
sometimes with more activity in one of the two. Four animals in the LDLD entrainment condition ( 2 in Expt 1 and 2 in Expt 2) maintained a unimodal pattern of wheel-running activity (i.e., they did not bifurcate), and were thus excluded from analyses. Mice in the Advancing group in Experiment 1 exhibited advancing patterns of wheel-running activity, with high amounts of transient activity in the photophases, typical of exposure to a changing light/dark cycle (Fig 2C).

\subsection{Fear conditioning}

\subsubsection{Experiment 1}

Freezing during the five minutes of training reflects learning, or acquisition, of fear memory. In our protocol, tone-shock pairings occurred at minutes 3, 4 and 5. In Experiment 1, a Two-way Repeated Measures ANOVA with Minutes and Entrainment State as factors and sex as a covariate revealed that Control, Bifurcated and Advancing animals demonstrated acquisition during training (RM ANOVA, $p<0.001$ for Minute) with no group differences across the five minutes of training ( $p=0.77$ for Group and $p=0.23$ for Minute $x$ Group Interaction; Fig 3A). Sex did not have any effect when other factors were considered, though there was a trend ( $p=0.09$ for Sex and $p=0.09$ for Minute $x$ Sex Interaction). There were no differences across the three groups in baseline locomotor activity (Fig 3B) or shock reactivity (Fig 3C; 2-way ANOVA for Group x Sex, $p<0.05$ for Sex in both measures; Group and Interactions for both measures, all $p$ values $>0.05$ ). In Experiment 1, there was no effect of group on post-shock freezing (One-Way ANOVA for Group with Sex as a covariate, $p=0.49$; Fig 3D). 
In Experiment 1, there were no significant differences in levels of freezing to context as a 5-min average by group (One-Way ANOVA with Sex as a covariate, $p=0.72$; Fig 4E). However, there was an effect of group on freezing to the tone (average freezing during the 330 -second tone presentations) (One-Way ANOVA with Sex as a covariate, $p<0.05$ ); post-hoc t-tests indicate animals that were chronically shifted (Advancing) showed impaired memory for tone compared to both Control $(p<0.05)$ and Bifurcated animals $(p<0.05$; Fig 4F).

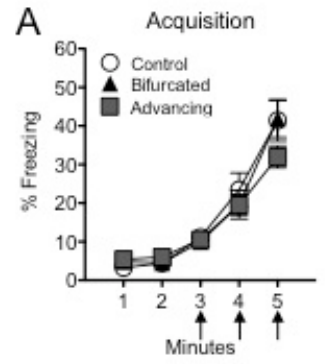

D

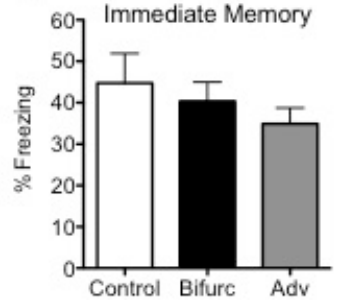

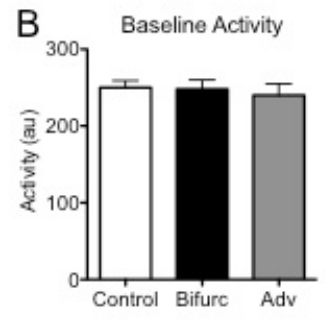

E

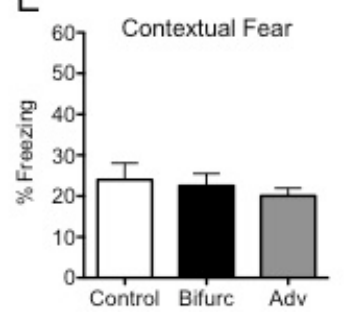

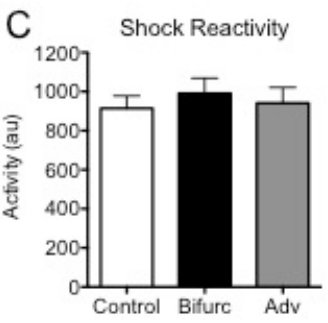

$\mathrm{F}$

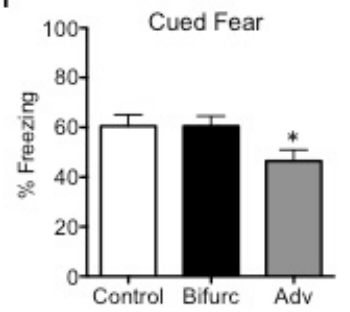

Figure 3. Circadian disruption, but not bifurcation, impairs retrieval (Experiment 1). Acquisition is shown as percent (\%) freezing over minutes 1 through 5 of training (A). Clear circles, black triangles, and gray squares represent the control, bifurcated, and advancing groups, respectively. Arrows indicate the administration of tone-shock pairings at minutes 3, 4 and 5. In all bar graphs for Experiment 1, clear bars represent the control group, filled bars represent the bifurcated group, and gray bars represent the advancing group. There were no differences across the three groups in baseline locomotor activity (B) or shock reactivity (C) during acquisition. Post-shock freezing and retrieval for both context and tone are shown as percent (\%) freezing. Post-shock freezing is measured here as the percent freezing in the five-minute period that immediately follows the five-minute training session (D). There were no differences between groups for either post-shock freezing (D) or for contextual fear $(E)$. The advancing group froze less in response to the tone presentation than both the controls and the bifurcated animals (F). There were no group differences between groups in acquisition. 


\subsubsection{Experiment 2}

In Experiment 2, a two-way repeated measures ANOVA with Minute, Training Phase and Entrainment State as factors demonstrated that Bifurcated animals exhibited a small but significant decrement in acquisition compared to the Control groups. While all groups showed increased freezing across subsequent tone-shock pairings ( $p<0.0001$ for Minute), Bifurcated animals showed significantly lower rates of freezing during acquisition than both control groups in Minutes 4 and 5 of training $(p<0.001$ for Entrainment State, and $p<0.001$ for Minute $x$ Entrainment State interaction; Bonferroni-adjusted post-hoc tests for Minutes 4 and 5 significant at $p<0.05$; Fig $4 \mathrm{~A}$ ). Control animals trained in the PM showed significantly higher rates of freezing during acquisition compared to Control animals trained in the AM and to Bifurcated animals trained at either phase $(p<0.05$ for Phase, but $p=0.16$ for Minute $\times$ Phase; Bonferroni-adjusted post-hoc tests for Minute 3 for PM Controls vs AM Controls and Bifurcated groups all significant at $p<0.05$ or less). There was no significant interaction of Minute $x$ Training Time $x$ Entrainment State $(p=0.25)$, or for Entrainment State $x$ Training Time $(p=0.09)$. There were no differences across the four groups in baseline locomotor activity or shock reactivity (not shown; both $p$ values $>0.05$ ) For post-shock freezing in Experiment 2, a two-way ANOVA with Entrainment State and Training Phase as factors yielded a main effect of Phase $(p<0.05), a$ trend for Entrainment State $(p=0.07)$ and no significant interaction $(p=0.42$; Figure 4B). Interestingly, post-hoc $t$-tests (planned comparisons) reveal an effect 
of Phase for Control animals (AM-trained control animals showed lower rates of freezing during the post-shock freezing test compared to animals trained at the PM phase (t-test, $p<0.05)$, while Bifurcated animals trained $12 \mathrm{~h}$ apart did not (ttest, $p=0.37)$. Taken together, the acquisition and post-shock freezing data appear to indicate that the two subjective days per $24 \mathrm{~h}$ that result from bifurcation may not differ from one another in terms of their effects on acquisition of conditioned fear.

In Experiment 2, bifurcated animals again show unimpaired retrieval of conditioned contextual and cued fear in mice relative to long day controls. To control for the significant difference in phase of training $\left(\mathrm{AM}_{1} \vee A M_{2}\right)$ for control groups tested for context at 12 , but not $24 \mathrm{~h}$ post-training, we examined the effect of entrainment state in groups tested $24 \mathrm{~h}$ later only. For groups tested $24 \mathrm{~h}$ posttraining, there was no significant effect of entrainment state on context $(p=0.09$, Fig 4C) or cued retrieval ( $p=0.19$, Fig 4D).

In addition to the phase effects found in control animals for acquisition and post-shock freezing, phase of training had an effect on retrieval as well. AMtrained Control animals showed impaired retrieval for context compared to animals trained at the PM phase when the train-test interval was $12 \mathrm{~h}(p<0.05)$, but not when it was $24 \mathrm{~h}(p=0.86$; Fig 4E). There were no phase differences in retrieval for tone (all $p$ values $>0.05$; Fig $4 \mathrm{~F}$ ). Unlike Controls, Bifurcated groups trained in $\mathrm{AM}_{1}$ did not differ from those trained in $\mathrm{AM}_{2}$ in expression of conditioned contextual or cued fear when tested $12\left(\mathrm{AM}_{1} \vee \mathrm{AM}_{2} t\right.$-test for context, 
$p=0.71$, Fig $4 \mathrm{G}$; for tone, $p=0.32$, Fig $4 \mathrm{H})$ or $24 \mathrm{~h}\left(\mathrm{AM}_{1} \vee \mathrm{AM}_{2} t\right.$-test for context, $p=0.32$, Fig $4 \mathrm{G}$; for tone, $p=0.49$, Fig $4 \mathrm{H}$ ) post-training.

Contrary to previous reports ${ }^{15-17}$, retrieval did not change over time in Control animals trained in the PM (PM 12 v 24 t-test for context, $p=0.92$, Fig 4E; tone, $p=0.44$; Fig 4F), though it did for context in Control AM mice (AM $12 \vee 24$, context, $p<0.05$, tone, $p=0.06$; Fig $4 \mathrm{E}$ ). Retrieval did not change over time for Bifurcated animals $\left(\mathrm{AM}_{1} 12 \vee 24\right.$ t-test for context, $p=0.79$, Fig $4 \mathrm{G}$; tone, $p=0.28$,

Fig 4H; $\mathrm{AM}_{2} 12$ v 24 t-test for context, $p=0.28$, Fig 4G; tone, $p=0.54$, Fig $4 \mathrm{H}$ ).
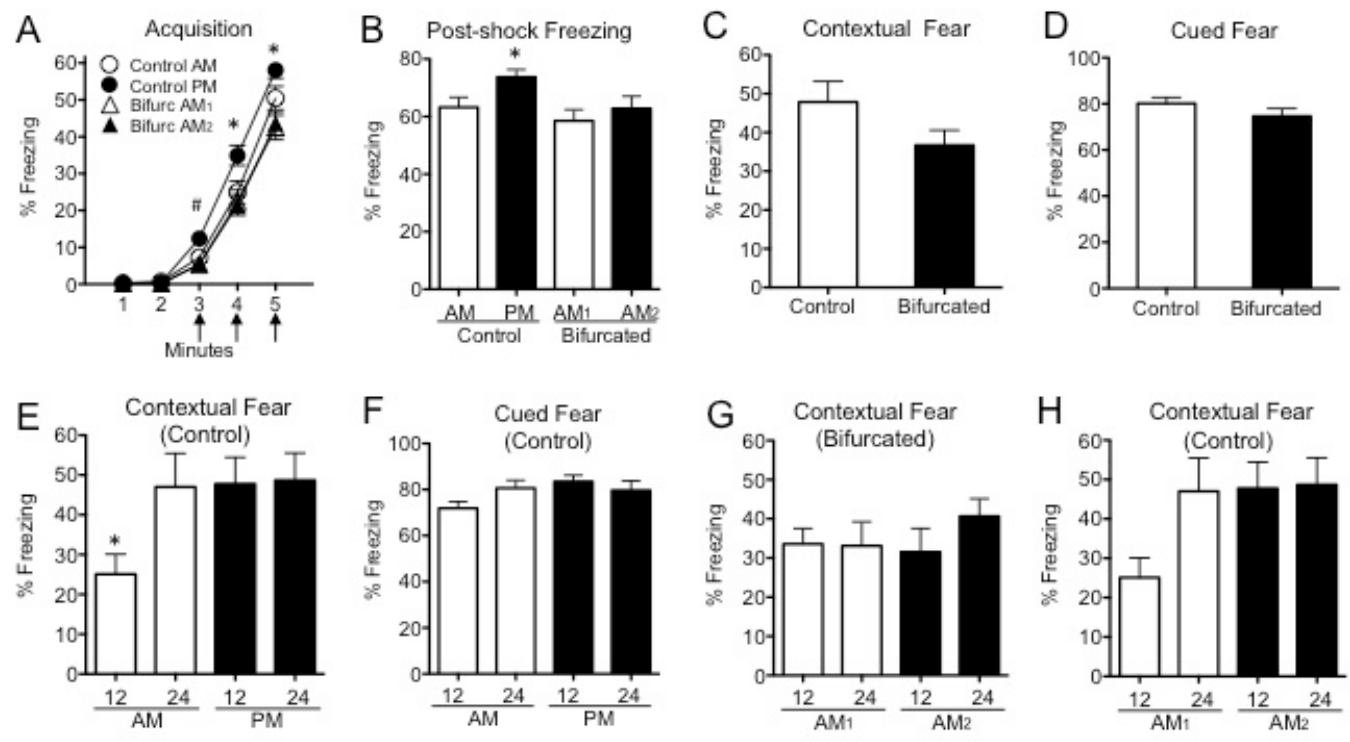

Figure 4. Phase and interval effects are found for control, but not bifurcated, animals (Experiment 2). Acquisition is shown as percent (\%) freezing over minutes 1 through 5 of training $(A)$. Groups trained in the morning $\left(A M\right.$ and $A M_{1}$ ) are represented by clear symbols, while groups trained $12 \mathrm{~h}$ later $\left(\mathrm{AM}_{2}\right)$ are represented by black symbols (circles for control groups, and triangles 
for bifurcated groups). Bifurcated animals showed significantly lower rates of freezing during acquisition than both control groups in Minutes 4 and 5 of training, indicated by the symbol $\left({ }^{\star}\right)$. Control animals trained in the PM showed significantly higher rates of freezing during Minute 3 of acquisition than both control animals trained in the AM and than bifurcated animals trained at either phase, indicated by the symbol (\#). Phase effects were found in post-shock freezing for control, but not bifurcated, animals (B). Post-hoc t-tests indicate significantly higher freezing in the PM group relative to the other 3 groups $(p s<0.5)$, indicated by a $\left(^{*}\right)$. For each entrainment state, data from both the $24 \mathrm{~h}$ interval groups were combined to directly compare controls to bifurcated animals over retrieval measures. No group differences were found for either context (C) or cued (D) fear. When phase and interval were examined, control animals trained in the AM showed less contextual freezing after a $12 \mathrm{~h}$ train-test interval than a $24 \mathrm{~h}$ one, and less than those trained in the PM phase, irrespective of interval $(E)$, indicated by the symbol $\left(^{*}\right)$. No phase or interval effects were found for cued fear in control animals (F), nor were any found for bifurcated animals for either form of memory $(\mathrm{G}$ and $\mathrm{H})$.

\section{Discussion}

5.1 Bifurcation does not disrupt long-term memory to the extent of a jet-lag paradigm.

In Experiment 2, bifurcated animals showed a small but significant decrement in acquisition and post-shock freezing compared to control animals. Despite this, learning is intact in bifurcated animals, as shown by the increased freezing over Minutes 3 through 5. Additionally, they show no impairment in retrieval for either contextual or cued fear compared to control animals in either Experiment 1 or Experiment 2. The implication of this is that the reorganization of the SCN that takes place under a bifurcated entrainment state need not disrupt learning and memory. Despite poorer acquisition (and presumably encoding), deficits observed in acquisition and post-shock freezing did not persist into longterm retrieval. Bifurcation may thus protect against negative effects of phase in context fear observed in the control group (Figs 4A and 4E, discussed below). 
Post-shock freezing in our protocol is the level of freezing during the five minute period without stimuli that immediately follows training. It is less well understood than other learning measures but is thought to reflect immediate memory for the association between the context and shock ${ }^{40,41}$. While it may have been informative to assess short-term memory at a more standard, 1 or $2 \mathrm{~h}$ posttraining, our protocol was carefully chosen to maximize the ability to test retrieval after relatively short intervals (12 to $24 \mathrm{~h}$ post-training) while minimizing additional testing that may have compromised behavioral entrainment.

By contrast, animals that were chronically shifted (Experiment 1), while trained and tested at comparable phases of the LD cycle, showed impaired memory for tone compared to both control and bifurcated animals (Fig 3F). It is possible that the decreased freezing to the tone presentation observed in the advancing group reflects not a memory deficit per se, but a small deficit in acquisition, which was observed but did not reach significance in our protocol. Other, more sensitive measures may be needed to differentiate between the two possibilities. Previous studies have found that while circadian phase affects training and retrieval of contextual fear in C57BL/6 mice, tone memory appears to be independent of circadian phase in some ${ }^{42}$, but not all ${ }^{15}$, circadian perturbation paradigms. Therefore, it is probable that the deficit for retrieval of cued memory seen in the Advancing group is a direct consequence of circadian disruption, rather than an effect of testing at a non-comparable phase. Bifurcated animals freeze to the tone at rates comparable to control animals and higher than the chronically-advanced mice (Fig 3F). This suggests that the steady-state 
reorganization of the circadian system in bifurcated mice does not disrupt retrieval to the extent of a jet-lag paradigm.

5.2 Phase effects are found for control, but not bifurcated, mice. In our study, control animals (12:12) trained during the transition from night to day showed impaired acquisition, post-shock freezing, and retrieval for context $12 \mathrm{~h}$ post-training compared to control animals trained during the transition into night. Like other studies, we find phase effects in our control mice for phase of training and testing when holding the train-test interval constant. As in Chaudhury and Colwell ${ }^{15}$, phase affected rates of freezing during acquisition (Fig 4A) and contextual retrieval (Fig 4E), though we observed no such difference in cued retrieval (Fig 4F). Similarly, as in other studies ${ }^{15,16,18}$, our results support evidence for a "time stamp" for learning, wherein retrieval is higher when training and testing occur at the same time of day (Fig 4E). However, our findings differ from these studies in a number of ways. In Cai et al. ${ }^{16}$, higher rates of freezing $12 \mathrm{~h}$ post-training were seen in 129B6 mice trained before subjective day, and not night. Similarly, Chaudhury and Colwell ${ }^{15}$ reported higher rates of freezing in $\mathrm{C}-3 \mathrm{H}$ and $\mathrm{C} 57 \mathrm{BL} / \mathrm{J} 6$ mice when training and testing occur during subjective day. These differences may be attributable to differences in strain, fear conditioning protocol (i.e., time between context and tone tests; the number, timing, or voltage of shocks), or to the fact that our mice had access to running wheels. In a report by Valentinuzzi and colleagues ${ }^{42}$ wherein phase of fear conditioning was examined in C57BL/6J mice with access to running wheels, animals trained and 
tested early in subjective night, rather than day, showed higher rates of freezing to context $24 \mathrm{~h}$ post-training. In rats, performance on a novel location recognition task, which like the context test in our paradigm is hippocampal-dependent, likewise peaked at night rather than day ${ }^{43}$. Finally, as with any study of daily learning patterns, it is a possibility that freezing levels could be influenced by daily rhythms in locomotor activity as well as by phase or interval effects on learning, per se. Despite efforts to control for such effects by training and testing during light transitions, we cannot exclude the possibility, for example, that the transition to subjective night may have attenuated freezing in the PM 12 group in Experiment \#2. The use of additional learning and memory models could help unconfound such possible influences.

We found no evidence that the two subjective days per $24 \mathrm{~h}$ seen in bifurcated animals differentially affect acquisition or consolidation of conditioned fear; bifurcated animals trained $12 \mathrm{~h}$ apart showed no differences in acquisition, post-shock freezing, or retrieval for context or tone (Experiment 2). These findings do not exclude a phase dependency that could be detected with more frequent sampling (i.e., every $6 \mathrm{~h}$ instead of 12). The effect of training and testing bifurcated animals before subjective day vs night was never explicitly examined in these experiments: In Experiment 2, all bifurcated animals, whether trained in $A M_{1}$ or $A M_{2}$, were trained during the transition to a photophase, whereas in Experiment 1, training and testing always occurred during the transition to a scotophase. 
5.3 Interval effects were found for control mice, but not bifurcated mice.

In previous work, animals with only a $12 \mathrm{~h}$ subjective night between training and test showed a retrieval decrement compared to other groups ${ }^{16}$. While this same pattern was not observed in the present results, we demonstrated a different interaction between phase and interval (Fig 4E). By convention, we have induced bifurcation by introducing the animals to a novel wheel at the start of one of the scotophases ${ }^{2}$, and therefore wheels were used in our protocol. Running wheels have been shown to change the organization of sleep (Welsh et al., 1988), increase learning ${ }^{44,45}$ and synaptic plasticity ${ }^{46}$, and rescue induced learning deficits ${ }^{47,48}$. While it is unknown whether the wheels may have compensated for small decrements dependent on sleep or other variables, the fact that our protocol was sensitive to differences in circadian manipulation (Experiment 1) and phase (Experiment 2) in control animals indicates that the wheels did not create a general ceiling effect. Further, $12: 12$ and 6:6:6:6 conditions were chosen so that total light exposure over $24 \mathrm{~h}$ cycle remained the same for LD controls and LDLD groups at time of training and test. As the two week baseline photoperiod for LDLD animals (18:6) differed from those of the controls, we cannot we cannot rule out the possibility that the extended photoperiod may have exerted additional effects on the animals which may have affected subsequent learning and memory.

\section{Conclusions}


Despite a major reorganization of the circadian timing system, acquisition and consolidation of memory is intact in bifurcated animals. While control animals have a slight significant advantage in acquisition and post-shock freezing over bifurcated animals trained during the transition to a photophase (Experiment 2), this advantage does not persist through retrieval of the memory, and is not present when bifurcated animals are trained and tested during the transition to a scotophase (Experiment 1). The lack of a phase effect in bifurcated animals in Experiment 2 suggests that $A M_{2}$ and $A M_{2}$ training, while $12 \mathrm{~h}$ apart, most likely occurred at a functionally equivalent circadian phase in bifurcated animals. Therefore, in terms of acquisition and retrieval of conditioned fear, each subjective day and each subjective night may be equivalent in these mice. In sum, it appears that the memory for conditioned fear in bifurcated mice is intact and superior to that observed in mice undergoing a simulated jet-lag paradigm. Therefore, unlike effects of chronic jet-lag, dissociation of oscillatory circadian components may not impair retrieval per se. These results have implications for understanding the organization and flexibility of the circadian system, and do not preclude bifurcation as a potential model for application in human shift-work.

\section{Acknowledgements}

The authors would like to thank Antonio Mora and Gil Sanchez for their excellent animal care. Additional thanks go to Qays Poonawala, Jeremy Johnson, Matthew Hermann and Deborah May for their technical assistance. This work was 
supported by the National Institutes of Health (R01 HD036460) and the Office of

Naval Research (N000141310285).

References1. Evans J, Davidson AJ. Health consequences of circadian disruption in humans and animal models. Prog Mol Biol Trans/ Sci. 2013;119:283-323.

2. Gorman MR, Elliott JA. Entrainment of 2 subjective nights by daily light:dark:light:dark cycles in 3 rodent species. J Biol Rhythms. 2003;18(6):502-512. doi:10.1177/0748730403260219.

3. Raiewski EE, Elliott JA, Evans JA, Glickman GL, Gorman MR. Twice daily melatonin peaks in Siberian but not Syrian hamsters under $24 \mathrm{~h}$ light:dark:light:dark cycles. Chronobiol Int. 2012:1-10. doi:10.3109/07420528.2012.719965.

4. Yan L, Silver R, Gorman M. Reorganization of suprachiasmatic nucleus networks under 24-h LDLD conditions. J Biol Rhythms. 2010;25(1):19-27. doi:10.1177/0748730409352054.

5. Watanabe T, Naito E, Nakao N, Tei H, Yoshimura T, Ebihara S. Bimodal clock gene expression in mouse suprachiasmatic nucleus and peripheral tissues under a 7-hour light and 5-hour dark schedule. J Biol Rhythms. 2007;22:58-68. doi:10.1177/0748730406295435.

6. Gorman MR, Steele NA. Phase angle difference alters coupling relations of functionally distinct circadian oscillators revealed by rhythm splitting. J Biol Rhythm. 2006;21:195-205.

7. Harrison EM, Gorman MR. Changing the waveform of circadian rhythms: considerations for shift-work. Front Neurol. 2012;3(72):1-7. doi:10.3389/fneur.2012.00072.

8. Harrison EM, Gorman MR. Rapid adjustment of circadian clocks to simulated travel to time zones across the globe. J Biol Rhythms. 2015. doi:10.1177/0748730415598875.

9. Evans JA, Elliott JA, Gorman MR. Dynamic interactions between coupled oscillators within the hamster circadian pacemaker. Behav Neurosci. 2010;124(1):87-96.

10. Anagnostaras SG, Gale GD, Fanselow MS. Hippocampus and contextual fear conditioning: Recent controversies and advances. Hippocampus. 2001;11:8-17.

11. Anagnostaras SG, Maren S, Fanselow MS. Temporally graded retrograde amnesia of contextual fear after hippocampal damage in rats: Withinsubjects examination. J Neurosci. 1999;19(3):1106-1114.

12. Gale GD, Anagnostaras SG, Godsil BP, et al. Role of the basolateral amygdala in the storage of fear memories across the adult lifetime of rats. J Neurosci. 2004;24(15):3810-3815. doi:10.1523/JNEUROSCI.410003.2004.

13. Gerstner JR, Lyons LC, Wright KPJ, Loh DH, Rawashdeh O, Eckel-mahan 
KL. Cycling behavior and memory formation. J Neurosci.

2009;29(41):12824-12830.

14. Wright KP, Lowry CA, LeBourgeois MK. Circadian and wakefulness-sleep modulation of cognition in humans. Front Mol Neurosci. 2012;5(50):1-12. doi:10.3389/fnmol.2012.00050.

15. Chaudhury D, Colwell CS. Circadian modulation of learning and memory in fear-conditioned mice. Behav Brain Res. 2002;133(1):95-108. doi:10.1016/S0166-4328(01)00471-5.

16. Cai DJ, Shuman T, Gorman MR, Sage JR, Anagnostaras SG. Sleep selectively enhances hippocampus-dependent memory in mice. Behav Neurosci. 2009;123(4):713. doi:10.1037/a0016415.

17. Loh DH, Navarro J, Hagopian A, Wang LM, Deboer T, Colwell CS. Rapid changes in the light/dark cycle disrupt memory of conditioned fear in mice. PLoS One. 2010;5(9). doi:10.1371/journal.pone.0012546.

18. Ralph MR, $\mathrm{Ko} \mathrm{CH}$, Antoniadis EA, et al. The significance of circadian phase for performance on a reward- based learning task in hamsters. Behav Brain Res. 2002;136:179-184.

19. Nakatsuka H, Natsume K. Circadian rhythm modulates long-term potentiation induced at CA1 in rat hippocampal slices. Neurosci Res. 2014;80:1-9.

20. Chaudhury D, Wang LM, Colwell CS. Circadian regulation of hippocampal long-term potentiation. J Biol Rhythms. 2008;20(3):225-236. doi:10.1177/0748730405276352.Circadian.

21. Legates TA, Altimus $\mathrm{CM}$, Wang $\mathrm{H}$, et al. Aberrant light directly impairs mood and learning through melanopsin-expressing neurons. Nature. 2012;491:594-598. doi:10.1038/nature11673.

22. Craig LA, Mcdonald RJ. Chronic disruption of circadian rhythms impairs hippocampal memory in the rat. Brain Res Bull. 2008;76:141-151. doi:10.1016/j.brainresbull.2008.02.013.

23. Neto SPD, Carneiro BTS, Valentinuzzi VS, Araujo JF. Dissociation of the circadian rhythm of locomotor activity in a $22 \mathrm{~h}$ light-dark cycle impairs passive avoidance but not object recognition memory in rats. Physiol Behav. 2008;94:523-527. doi:10.1016/j.physbeh.2008.03.013.

24. Devan BD, Goad EH, Petri HL, et al. Circadian phase-shifted rats show normal acquisition but impaired long-term retention of place information in the water task. Neurobiol Learn Mem. 2001;75(1):51-62. doi:10.1006/nIme.1999.3957.

25. Fernandez F, Lu D, Ha P, et al. Dysrhythmia in the suprachiasmatic nucleus inhibits memory processing. Science (80- ). 2014;346(6211):854857. doi:10.1126/science.1259652.

26. Ruby NF, Fernandez F, Garrett A, et al. Spatial memory and long-term object recognition are impaired by circadian arrhythmia and restored by the GABA antagonist pentylenetetrazole. PLoS One. 2013;8(8):e72433. doi:10.1371/journal.pone.0072433.

27. Gibson EM, Wang C, Tjho S, Khattar N, Kriegsfeld LJ. Experimental "jetlag" inhibits adult neurogenesis and produces long-term cognitive 
deficits in female hamsters. PLoS One. 2010;5(12):e15267.

28. Kott J, Leach G, Yan L. Direction-dependent effects of chronic "jet-lag" on hippocampal neurogenesis. Neurosci Lett. 2012;515:177-180. doi:10.1016/j.neulet.2012.03.048.

29. Cai DJ, Shuman T, Harrison EM, Sage JR, Anagnostaras SG. Sleep deprivation and Pavlovian fear conditioning. Learn Mem. 2009;16(10):595599. doi:10.1101/lm.1515609.

30. Hagewoud R, Whitcomb SN, Heeringa AN, Havekes R, Koolhaas JM, Meerlo P. A time for learning and a time for sleep: The effect of sleep deprivation on contextual fear conditioning at different times of the day. Sleep. 2010;33(10):2-9.

31. Kohman RA, Clark PJ, Deyoung EK, Bhattacharya TK, Venghaus CE, Rhodes JS. Voluntary wheel running enhances contextual but not trace fear conditioning. Behav Brain Res. 2012;226:1-7. doi:10.1016/j.bbr.2011.08.031.

32. Eisenberg DP, Kohn PD, Baller EB, Bronstein J a, Masdeu JC, Berman KF. Seasonal effects on human striatal presynaptic dopamine synthesis. $J$ Neurosci. 2010;30(44):14691-14694. doi:10.1523/JNEUROSCI.195310.2010.

33. Prendergast $B$, Nelson $R$. Affective responses to changes in day length in Siberian hamsters. Psychoneuroendocrinology. 2005;30:438-452.

34. Prendergast BJ. Behavioral tolerance to endotoxin is enhanced by adaptation to winter photoperiods. Psychoneuroendocrinology. 2008;33(4):540-545. doi:10.1016/j.psyneuen.2007.12.008.

35. Walton JC, Haim A, Spieldenner JM, Nelson RJ. Photoperiod alters fear responses and basolateral amygdala neuronal spine density in whitefooted mice (Peromyscus leucopus). Behav Brain Res. 2012;233:345-350.

36. Gorman MR, Elliott JA, Evans JA. Plasticity of hamster circadian entrainment patterns depends on light intensity. Chronobiol Int. 2003;20(2):233-248. doi:10.1081/CBI-120018576.

37. Gorman MR, Lee TM. Daily novel wheel running reorganizes and splits hamster circadian activity rhythms. J Biol Rhythms. 2001;16(6):541-551. doi:10.1177/074873001129002231.

38. Carmack SA, Howell KK, Rasaei K, Reas ET, Anagnostaras SG. Animal model of methylphenidate's long-term memory-enhancing effects. Learn Mem. 2014;21(2):82-89. doi:10.1101/lm.033613.113.

39. Anagnostaras SG, Wood SC, Shuman T, et al. Automated assessment of pavlovian conditioned freezing and shock reactivity in mice using the video freeze system. Front Behav Neurosci. 2010;4(September):1-11. doi:10.3389/fnbeh.2010.00158.

40. Fanselow M. Associative vs Topographical Accounts of the immediate Shock-Freezing Deficit in Rats : Implications for the Response Selection Rules Governing Species- Specific Defensive Reactions. Learn Motiv. 1986;17:16-39.

41. Wood SC, Anagnostaras SG. Interdependence of measures in Pavlovian conditioned freezing. Neurosci Lett. 2011;505(2):134-139. 
doi:10.1016/j.neulet.2011.10.006.

42. Valentinuzzi V, Kolker D, Vitaterna M, Ferrari E, Takahashi J, Turek F. Effect of circadian phase on context and cued fear conditioning in C57BL/6J mice. Anim Learn Behav. 2001;29(2):133-142.

43. Takahashi Y, Sawa K, Okada T. The diurnal variation of performance of the novel location recognition task in male rats. Behav Brain Res. 2013;256:488-493.

44. Greenwood BN, Strong P V, Foley TE, Fleshner M. A behavioral analysis of the impact of voluntary physcial activity on hippocampus-dependent contextual conditioning. Hippocampus. 2009;19(10):988-1001. doi:10.1002/hipo.20534.A.

45. Kohman RA, Clark PJ, DeYoung EK, Bhattacharya TK, Venghaus CE, Rhodes JS. Voluntary wheel running enhances contextual but not trace fear conditioning. Behav Brain Res. 2013;226(1):1-7. doi:10.1016/j.bbr.2011.08.031.VOLUNTARY.

46. Patten AR, Sickmann H, Hryciw BN. Long-term exercise is needed to enhance synaptic plasticity in the hippocampus. Learn Mem. 2013;20:642647. doi:10.1101/lm.030635.113.

47. Christie B, Swann S, Fox C, et al. Voluntary exercise rescues deficits in spatial memory and long-term potentiation in prenatal ethanol-exposed male rats. Eur J Neurosci. 2005;21(6):1719-1726.

48. Fardell J, Vardy J, Sha J, Johnston I. Cognitive impairments caused by oxaliplatin and 5-fluorouracil chemotherapy are ameliorated by physical activity. Psychopharmacology (Berl). 2011;220(1):183-193. 